\title{
Understanding Maturity of Collaborative Network Organizations by Using B-ITa Processes
}

\author{
Faiza Allah Bukhsh ${ }^{1}$, Maya Daneva $^{2}$, and Hans Weigand ${ }^{1}$ \\ 1 Department of Information Management, Tilburg University, P.O. Box 90153, \\ Tilburg, The Netherlands \\ \{f.a.bukhsh, h. weigand\}@uvt.nl \\ 2 Department of Computer Science, University of Twente, P.O. Box 21, 7500 AE \\ Enschede, The Netherlands \\ m.daneva@utwente.nl
}

\begin{abstract}
Organization's concerns as controlling costs, improving quality, increasing effectiveness, and managing risk increasingly impose strong requirements on Business-IT alignment (B-ITa). Several Maturity Models came into being for considering improvement actions in B-ITa. The IT-Enabled Collaborative Network organizations (ICoNOs) maturity model specifically addresses the processes needed for achieving B-ITa in networked organizations. We have evaluated ICoNOs maturity model on a case study in an Asian organization. On the basis of this study, we propose to introduce a new domain named as "Cost Management".
\end{abstract}

Keywords: Business-IT Alignment, Inter-organizational collaboration, Maturity Models, Case Study.

\section{Introduction}

In the recent years the development of Information Technology (IT) resulted in inventing a variety of collaboration and coordination models based entirely on inter-organizational systems. For communications and collaboration to add value to the business, businesses need to align their business and IT sides. This need is also very well recognized in inter-organizational settings, where multiple businesses or government organizations team up with each other to deliver together a product or a service to clients. In this paper, we adopt the following definition of a network organization: "A network organization is a situation that come up when independent people and groups, linked across boundaries, work together for achieving a common goal" 1419. The relationship of network organizations when studied in perspective of business-IT alignment (B-ITa) gives rise to the question of how to measure the maturity of the relationship. To respond to this, researchers have come up with a variety of maturity models (MM) [17151824]. In this research, we focus on one most recent MM, namely ICoNOs [21] developed by Santana Tapia [20]. This model has been evaluated by its author in terms of its suitability on European and American CNOs. However, the previously published evaluation studies are preliminary only and Santana Tapia [20] acknowledged that more evaluation research is needed to confirm the suitability and the 
usefulness of the ICoNOs model for both government and non-government NOs. In this paper we will take into account the challenge to evaluate the suitability of ICoNOs in a variety of settings, to derive lessons learnt from evaluation study, and to use the learning to enhance the ICoNOs model.

Our paper builds upon the knowledge gained in the early evaluation studies, and sets out to apply the model to a case study in Asia and reflect on the experiences. We carry out an evaluation study in which we attempt to answer the following Research Question: What is the suitability of the ICoNOs MM to contexts of government Network organizations/Collaborative Network Organizations (NOs/CNOs) in developing countries for assessing their B-ITa.

In order to justify the selection of ICoNOs MM in section 2 we have presented a literature review. For evaluating the ICoNOs MM we have selected the qualitative research approach 28. To do the evaluation, we have selected an Interview based approach, in which we are following a research cycle [31] [32] presented in section 3. Section 4 closes with conclusion and some future research directions.

\section{Background}

MMs describe evolution of an entity (Organization/ NOs/ CNOs etc). These models are designed for the purpose of assessment and prediction of some attributes for an entity. Literature review done by Santana Tapia's is based on the models especially designed for the B-ITa [20]. In our literature review we explicitly included e-government and e-business models in which B-ITa is one of the key components (but not the only one).

Figure 1 show the literature review we have done on the MMs developed from 2007 till 2009 and e-government models developed from 2001 till 2008. In Santana Tapia's literature review whose scope ranged from 2001 till 2006, the author found that his surveyed MMs mostly relate to B-ITa in a single enterprise and few with B-ITa in CNOs. In case of CNOs the main focus is on processes

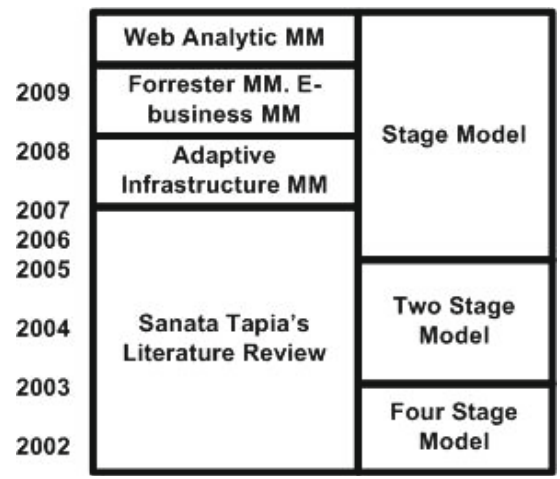

Fig. 1. Results at a Glance

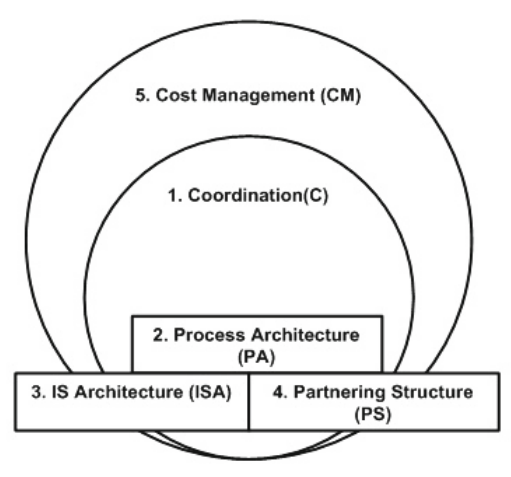

Fig. 2. Relationship of Cost Management with Other Domains 
and integration levels (such as SCM MM [15], E2A MM [24]), relationship (such as IT outsourcing MM [1]), IT sharing and information (such as in extended CMM [17 ). We have found that Adaptive infrastructure MM [1] developed especially for the data center management. The well known Forrester MM [18] model address SAAS context while the last model included in the survey from MM's perspective is web analytic MM [7] which describes the maturity of an organization through web analysis. Models reviewed in e-government domain are the stage models which studied e-government growth with respect to multiple stages. With the help of MM, entities become aware of their current state in different areas. Maturity models are usually level based. Domains and levels characterize each MM. Domains define the specific area or direction and levels define the improvement path.

\subsection{The ICoNOs Maturity Model}

In the following, we will summaries ICoNOs MM domains and levels.

ICoNO's Levels [23] ICoNOs MM consist of five levels, which are used to describe the improvement path for CNOs to achieve B-ITa. These levels are,

- Level 1: Incomplete. At this level processes for a B-ITa domain are not performed or partially performed.

- Level 2: Isolated. Processes for particular B-ITa domain are planned and executed at initial levels. But these processes are isolated not managed form CNOs perspective.

- Level 3: Standardized. Processes are heading for standardization of a particular B-ITa domain from a CNOs perspective.

- Level 4: Quantitative Managed. Processes about a particular B-ITa domain use statistical and other quantitative techniques for quality measurement.

- Level 5: Optimized. Processes are improved for B-ITa based on common causes and interests.

ICoNO's Domains [23] All the levels defined above are used on certain domain. A domain is a group of processes used to improve a particular area in CNOs. ICoNOs MM consist of four domains which are as follows:

- Partnering Structure: Defines where the work will be done and who will be involved. For CNOs it describes organization structure, work division, roles and responsibilities.

- IS Architecture: It is about information management function their relationship to each other and the environment. It also describes the principles, guiding the design, and evolution of CNOs.

- Process Architecture: Describe about all the individual and collaborative processes needed to reach a shared goal of CNOs.

- Coordination: It is about managing the interaction and work among the CNOs while considering the dependencies among the processes. 


\subsection{Comparing ICoNOs Maturity Model with the Previously Published Work}

We must note that the six models presented in Section 2, are all geared to the networked settings. When comparing ICoNOs and any of the six models, we observe that mappings can be found between the ICoNOs design elements and the design elements of the models in our review. For example, the Four Stage Model [12], the HP AIMM 1 and the Forrester model [18] suggest a strong emphasis on processes and integration levels, on relationships, on information sharing, as ICoNOs does. We are however able to point out to areas that fall beyond the scope of ICoNOs. These are: culture, cost management, quality assurance. We trace the inclusion of these areas back to the objectives with which the six models were created. We also think that those areas that are beyond the ICoNOs MM's scope, can be considered as candidates for inclusion in ICoNOs MM, if one considers investigating the possible ways to extend the ICoNOs MM. We make a note of this because it is well possible that replicated evaluations of ICoNOs MM suggest needs for extending it. If this happens, then a researcher would not only use his/her observations coming out of his/her evaluation of ICoNOs MM, but also the observations that are provided through this literature review. Indeed, in the rest of this paper, we will see how we identified the need for one specific enhancement to the original ICoNOs MM and how we responded to this need.

\section{Case Study}

We have used an interview-based approach for evaluation of ICoNOs MM on an Asian organization (OG).Where OG has one of the largest citizen database. We started by conducting the interviews and concluded that as OG is originally developed by its strategic partners, so the administration don't know the details of technical terminologies evolved behind it. The overall case study plan was inspired by Yin [28] and other researchers [11]. These steps involves,

1. Decide on the MM and Interviewees: We have selected ICoNOs MM as the model to evaluate and OG as an organization for selecting the people to interview.

2. Present the ICoNOs MM to Interviewees: We gave a presentation regarding the ICoNOs MM and describe about the five maturity levels and four domains of ICoNOs. Those are Partnering structure, IS architecture, Process architecture and Coordination.

3. Conduct the Interviews. For the better understanding and convenience of the professionals, most of the interviews were conducted in Urdu (Pakistan's National Language) and then translated into English. In the following section we will discuss the results concluded, We carried out semi-structured interviews based on the interviewing practices recommended in [27]. For the interviews, we used the questionnaire in Santana Tapia's thesis (see Appendix D in [20] ). We got an opportunity to meet five professionals. Four

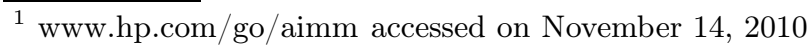


of them were from OG and one was from its local client. In OG our selected professionals were working on the national database of citizens and the one form its strategic client works on the passport authority. In this paper, instead of writing a detailed question-answer-session of each professional, we are dividing the interviews into three parts based on our three broad questions. Below, each question, the views of each interviewee are presented.

\subsection{The Data Collection}

Below we present samples from our interview data that we collected through our participants. This is to illustrate to the reader the kind of questions being asked, and the type of information being collected. In Section 3.2., we used this information to summarize our findings and draw some conclusions.

Question 1: How OG judges the strengths and weaknesses of the relationship with its partner organizations (namely, its clients)?

1st Interviewee: As OG is the Pakistan's largest database for the citizen, from the start of partnership all the clients change themselves according to OG. If there is some platform incompatibility then it is obligatory for client to adjust with OG. 2nd Interviewee: OG is the sole provider of citizen's data to other authorities so it has no competitor. Therefore clients have to make the effort to adjust with $\mathrm{OG}$.

3rd Interviewee: OG considers its strong and weak clients by considering the level of coordination that they have among them.

4th Interviewee: Capability of data exchange is the basis for the strong and weak relationship with its clients. For data exchange basic infrastructure needs to be the same. For example, in case of OG and it clients.

5th Interviewee: The requirement document provided by $\mathrm{OG}$ to its client is in fact the base of the judgment, for seeing the existing and future possibility of alignment between them.

Question 2: What are the aspects needed, for OG to work successfully with its clients?

1st Interviewee: The coordination on the 'architectural information' is required data.

2nd Interviewee: Cost considerations and quality control are the building stone of the successful relationship. As there exist no competitors for OG they can demand whatever they want.

3rd Interviewee: Coordination and communication among the clients and the $\mathrm{OG}$ is one of the major aspects to consider. Lack of communication produces a different outcome then expected or required.

4th Interviewee: Process architecture and business rules and data flows are the most important aspect for OG's success.

5th Interviewee: There exist many aspects but the most important among them are cost management and coordination.

Question 3: Do these recommendations (ICoNOs Maturity Model Domains) suits to $O G$ ? 
We make the note that the names of the ICoNOs MM domains were not provided in the orientation presentation explicitly, because we wanted the interviewees to give us the necessary domain names from OG themselves. We wanted to see whether the domain names and the earnings that would come up in the minds of the professionals, when thinking about B-ITa, would converge with or diverge from the original names and meanings used in the ICoNOs MM.

1st Interviewee: Maturity consideration with respect to coordination suits to OG but IS architecture and Process architecture are not very important. So we can say that among ICoNOs MM domain coordination and partnership structure (partnering structure in ICoNOs terminology) are basic recommendations.

2nd Interviewee: To measure the maturity of the $\mathrm{OG}$ with its clients recommendations such as coordination, partnership structure, IS and business process architecture are important but there should also be some cost consideration.

3rd Interviewee: So far there was no way by which OG can consider maturity aspect and these recommendations provide very practical and convenient framework.

4th Interviewee: Yes, they suit to OG.

5th Interviewee: Not only these recommendations suit to OG but they are also helpful for the clients. Because in this way the client can also find out at which level of maturity he is with OG or with any other organization.

In the following section we will conclude these answers.

\subsection{The Results}

In our case study research, we came across with following important findings,

- Coordination is fundamental among all the dimensions; we found that any other dimension like Partnering Structure, IS architecture or Process architecture are related to the Coordination. We can say that all the other three domains lie under the cover of Coordination.

- IS architecture and process architecture are being used but at OG, both architectures didn't have any distinct boundaries, in-fact they have overlapping boundaries.

There exist different terms but have same meaning. For example Partnering Structure is equivalent to Partnership Structure, Partnership, CNO is Network Organizations, Partnering Organizations. Based on our learning we confirm the presence of same four key domains (Partnering Structure, IS architecture, Process architecture and Coordination)proposed by Santana Tapia [22]: CNOs involved in B-ITa projects consider these domains for understanding B-ITa maturity. During the interviews three out of four interviews mention additional domains , in particular "Cost Management". But the most important are the four discussed by the ICoNOs MM. During our case study analysis we have considered validity threats states as 'single case study results cannot be generalized to update the ICoNOs MM model'. To confront it, our argument is that not 
only our case study results favor the introduction of cost domain but the results obtained by Santana Tapia [20] already indicate the importance of cost domain.

\subsection{Evaluation of Validity Threats in Interview-based Case Study}

We have validated the ICoNOs MM on one of the government organization in Pakistan and found that the ICoNOs MM is equally suitable to Asian government organizations. It is possible to challenge our conclusions by asking a number of questions, which are as follows:

- Was the organization very much developed government organization and, thus, a typical for Asian countries?

- Will ICoNOs MM be equally applicable to other Asian CNOs?

- If we apply ICoNOs MM on other government organizations, will the results be same?

We address these questions in more detail. First, OG is a large provider of government solutions serving many Asian countries and its solution delivery processes are developed and elaborated in a way similar to a solution provider organization in the Western countries (e.g. US or the European countries). This might pose a threat to the generalizability of our results in the sense that they might be specific to large Asian CNOs where one much developed partner (in terms of organizational design) instills its discipline, processes and cooperation rules over its collaborators (in this case, its clients). However, we think that the impact of this threat is minimal as OG seems to be a typical organization in the Asian market of government system delivery. We did a search for evidence on the characteristics (in terms of collaboration) of Asian e-government projects as available in web sites of market research firms in Asia (e.g. Gartner Asia, eGovAsia - Www.egovasia.com), the United Nations [26] and the Economist magazine. Specifically, we looked into what kinds of partners are included in Asian vendor and partnership ecosystems. We found that in a number of Asian countries, e-government is built-up with the participation of large solution providers collaborating with country-specific government authorities. We also acknowledge that Asian countries have similar e-government demands and, as it is the case of $\mathrm{OG}$, it is well possible for a solution provider to have as clients the governments of several countries.

Second, we can say that we have selected OG for convenience (in a way randomly) and without any prior analysis. The author of this paper had a previous work relationship with OG and was employed in an internship in a Pakistani government organization. The author was, thus, familiar with the government processes in the country and in the business areas for which OG delivers IT solutions. We acknowledge that other researchers might choose other country's organizations for the reasons similar to ours.

Third, we make the note that the interviews were carried out and transcribed in Urdu language, and also analyzed by one researcher only (namely, the leading author of this paper). Two senior researchers participated in planning the case 
study, but because they were not familiar with Urdu, there was no way for them to get actively involved in the data analysis. This poses a threat to the accuracy of the data collected and the analysis as the interpretation of the author could have been different from what the interviewees actually wanted to say. This threat was mitigated by a checking step in which others asked questions and attempted to triangulate the data with the information from official documents partly available in English from the web sites of $\mathrm{OG}$ and its clients. This triangulation step strengthened the feeling that the level of bias of the first author is reduced.

\subsection{Implication of Results from OG: Introduction of the Cost Management Domain}

The recommendation obtained by the interviewed professionals from OG suggests that cost is an important factor to consider. Here we compare this finding with what is known in literature about the role of Cost Management in B/ITa, and we motivate why we decided to include it as a new domain into the ICoNOs model.

Indeed, when searching the literature on the role of cost consideration in BITa, one can deduce that many theories and models 24456101025], are cost based. The common argument all of these theories and models have is that due to B-ITa cost is being shared among the businesses. Furthermore, the literature survey done by Santana Tapia [20] provides the basis for thinking that cost management is an important dimension of B/ITa. The above findings and our own results motivated us to extend ICoNOs by including the Cost management domain. Our goal of introducing this domain is to improve the completeness and performance of ICoNOs MM. For the purpose of introducing this domain, we set forth a set of questions:

What will be the name of the new domain?

From interviews we have concluded that B-ITa is affected by cost. Among many terms used by OG (Financial Constraint, Cost effects, Cost management, Financial regulations) we have selected "Cost Management" as the name of this new domain [13]. We think that this choice is justified because we found it was intuitive not only to our case study participants but also it converged with the terminology used in the B-ITa literature.

Will the name of the ICoNOs MM be changed after introduction of new domain? Yes it will change; we will name it as Enhanced ICoNOs MM.

How to define this domain?

The Cost Management domain can be defined as "the discipline of ensuring IT is obtained in the most effective price - which does not necessarily mean cheapest so that the CNOs can understand the cost of its services and/or products (based on ITIL [10]).

Why is it necessary to introduce it? Cost constraints always affect the progress of an organization. Despite the fact that all the other domains help us to understand the B-ITa, if financial constraints/standards of the partnering organization are 
not comparable (w.r.t organization's size, type, financial state. etc) then the ICoNOs MM domain may not be enough to make the right decisions.

Will the ICoNOs MM domains be affected by the introduction of a new domain? We think that they will be affected partially. Coordination is the heart of the B-ITa, without it two CNOs can never be aligned. If two CNOs have different cost considerations then how the coordination is possible at equal level? For example, the domain of partnering structure incorporates all aspects related to "IT governance" too. There are always some costs of governance.

Information System architecture and process architecture are also two ICoNOs MM domains and these are partially dependent on cost. These two domains are in fact dependent on information and process flows, information and process flow are affected by cost. From the case study results we found the relationship between the existing domains and new domain. Figure 2 shows relationship of cost domain with ICoNOs MM domains.

We make a note that, in the Figure2, the relationships among the four ICoNOs MM's domain are borrowed from Santana Tapia's work [23]. We have introduced only the relationship of "Cost Management" domain with ICoNOs MM's domains. Our literature review lets us think that the effect of cost on domains would vary with respect to organization type. According to de Koning and van der Marck [4] the decision of making an investment in an organization is dependent on the organization type/strength/status/maturity. For NOs/CNOs cost is important but how much the cost is important for NOs/CNOs would dependent on this organization's business rules. For CNOs where profit and lost is being shared among the partnering organization [23, cost considerations become important automatically.

\subsection{Enhanced ICoNOs MM: Cost Management Domain}

Santana Tapia discusses the possibility of the introduction of a new domain in Section 10.3 of his dissertation [20]. ICoNOs MM when evaluated on OG leads us to conclude that "Cost Management" is an important domain.

The introduction of new domain transforms the ICoNOs MM into Enhanced ICoNOs MM. Each domain has its B-ITa processes derived from the theories and models [235169816]. From extensive literature review Santana Tapia selected a set of B-ITa processes that make up the four domains (PS, IS, PA, $\mathrm{CO}$ ) as shown in Figure 3. For the new domain we used the same literature to identify processes that belong to the Cost Management domain.

The underlined and bold process names show the Cost management domain processes in the modeling map. The following six B-ITa processes are introduced for "Cost Management" domain.

First column of the Table 1 shows the levels of B-ITa processes. It can be argued why we position one process at level 4 and other at level 2. However the decision for such a positioning is derived by the definition of each process. 


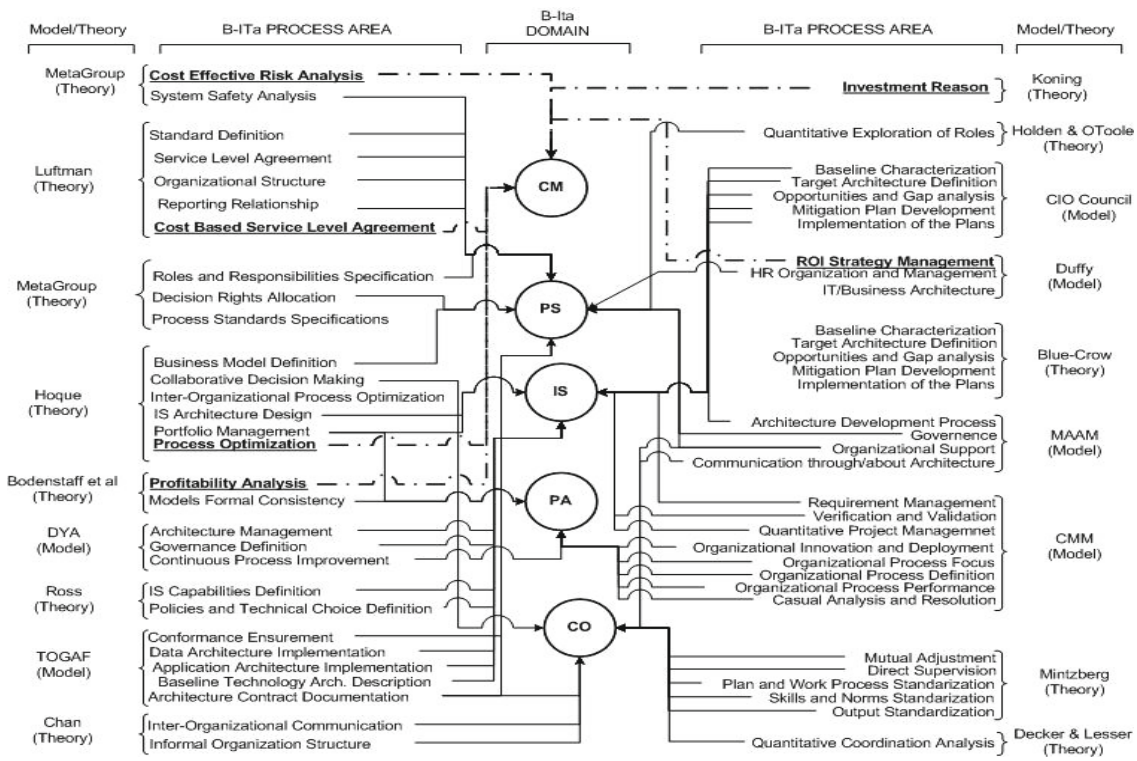

Fig. 3. B-ITa Processes

Table 1. Cost Management Processes

\begin{tabular}{|l|l|l|}
\hline Level & Process & Description \\
\hline 2 & $\begin{array}{l}\text { Cost-based service level } \\
\text { agreements [16] }\end{array}$ & $\begin{array}{l}\text { Describes cost-based cross-organizational ties for } \\
\text { decision-making and authority regulation, which in } \\
\text { result regulate the CNOs work. }\end{array}$ \\
\hline 3 & $\begin{array}{l}\text { ROI strategy and man- } \\
\text { agement formulation [5] }\end{array}$ & $\begin{array}{l}\text { Describes the financial management of the organization. } \\
\text { In this process, IT costs and benefits are part of general } \\
\text { costs and benefits. }\end{array}$ \\
\hline 4 & Process optimization [9] & $\begin{array}{l}\text { Describes the principles that support the alignment be- } \\
\text { tween business and IT by optimizing the cost related } \\
\text { business process. }\end{array}$ \\
\hline 4 & Profitability Analysis [2 & $\begin{array}{l}\text { Describes about how a CNO use the profit model in com- } \\
\text { bination with process model and event log to track a } \\
\text { snapshot to analyze the profitability of the CNO. }\end{array}$ \\
\hline 5 & $\begin{array}{l}\text { Investment } \\
\text { analysis } 4]\end{array}$ & $\begin{array}{l}\text { This process provides the knowledge to other parties that } \\
\text { are external to the CNO. For potential investors, it also } \\
\text { gives a reason to invest in the CNO. }\end{array}$ \\
\hline 5 & rest effective risk analy- & $\begin{array}{l}\text { Describes the sources of cost-based problems in business } \\
\text { also take the actions to prevent it in future. }\end{array}$ \\
\hline
\end{tabular}

\section{Conclusion}

Maturity Models seems to be a useful vehicle for understanding the alignment of the organization. To the best of our knowledge there exist no MM, which 
specifically discusses B-ITa in CNOs. The question raised in the Section 1 can be answered by saying that we found that the ICoNOs MM suits the context of Asian CNOs. We also found some similarities between Asian and Western CNOs context regarding the exposure of CNOs partners to disciplined processes. We proposed a new domain as an extension of the ICoNOs MM. It includes six B-ITa processes. Our decision to include these six processes is based on our literature analysis. However, we acknowledge that new scientific studies are being published each month on B-ITa and they may provide good argumentation for including new processes to our set of six. We therefore do not consider our Enhanced ICoNOs MM to be complete and we expect it be extended with more B-ITa processes whose evaluation may possibly add more precision into the final B-ITa maturity assessment itself. Follow-up qualitative case studies are also an important line of future research, so that the findings from literature are triangulated with insights from practitioners. This will build strength in the claim that Cost Management is an important domain to consider when assessing B-ITa maturity. We acknowledge that the maturity assessment is a labor-intensive and time-consuming process as it depends on the involvement of experts. We think that researchers can balance light-weight approaches to maturity data gathering and analysis with more 'interview based' approaches (e.g. interviews). What represents the right balance and how to achieve it remains an open question for the future.

\section{References}

1. Adelakun, O., Wabash, S.: IT outsourcing maturity model. In: Proceedings of the 13th European Conference on Information Systems, The European IS Profession in the Global Networking Environment, ECIS, vol. 4 (2004)

2. Bodenstaff, L., Wombacher, A., Reichert, M.: On formal consistency between value and coordination models. Technical Report Centre for Telematics and Information Technology University of Twente (2007)

3. CMMI Team: CMMI for development, version 1.2 (2006)

4. de Koning, D., van der Marck, P.: IT zonder hoofdpijn: een leidraad voor het verbeteren van de bedrijfsprestaties. Prentice Hall (2002)

5. Duffy, J.: Maturity models: Blueprints for e-volution. Strategy \& Leadership 29(6), 19-26 (2001)

6. Gunderson, S.: A review of organizational factors and maturity measures for system safety analysis. Systems Engineering 8(3), 234-244 (2005)

7. Hamel, S.: The web analytic maturity model (2009)

8. Henderson, J., Venkatraman, N.: Strategic alignment: Leveraging information technology for transforming organizations. IBM Systems Journal 32(1), 4-16 (1993)

9. Hoque, F.: The Alignment Effect: How to get real business value out of technology. FT Press (2002)

10. Janssen, P.: IT-servicemanagement volgens ITIL. Pearson Education (2005)

11. Johnson, B., Christensen, L.: Educational research: Quantitative, qualitative, and mixed approaches. Sage Publications, Inc. (2007)

12. Layne, K., Lee, J.: Developing fully functional e-government: A four stage model. Government Information Quarterly 18(2), 122-136 (2001) 
13. Lee, J.: The scientific endeavor (2000)

14. Lipnack, J., Stamps, J.: The teamnet factor: Bringing the power of boundary crossing into the heart of your business. Wiley (1993)

15. Lockamy III, A., McCormack, K.: The development of a supply chain management process maturity model using the concepts of business process orientation. Supply Chain Management: An International Journal 9(4), 272-278 (2004)

16. Luftman, J.: Assessing IT/business alignment. Information Systems Management 20(4), 9-15 (2003)

17. Ramasubbu, N., Krishnan, M., Kompalli, P.: Leveraging global resources: A process maturity framework for managing distributed development. IEEE Software 22(3), 80-86 (2005)

18. Ried, S., Rymer, J., Iqbal, R.: Forrester's SaaS maturity model: Transforming vendor strategy while managing customer expectations. Forrester Research (2008)

19. Santana Tapia, R.: What is a networked business? Technical Report Centre for Telematics and Information Technology, University of Twente (2006)

20. Santana Tapia, R.: Assessing business-IT alignment in networked organizations. PhD thesis. University of Twente (2009)

21. Santana Tapia, R.: Converging on business-IT alignment best practices: Lessons learned from a dutch cross-governmental partnership. In: ICE 2009: Collaborative Innovation: Emerging Technologies, Environments and Communities, Leiden, The Netherlands (2009)

22. Santana Tapia, R.: ICoNOs MM: The IT-enabled collaborative networked organizations maturity model. Leveraging Knowledge for Innovation in Collaborative Networks, 591-599 (2009)

23. Santana Tapia, R., Daneva, M., van Eck, P., Wieringa, R.: Towards a business-IT aligned maturity model for collaborative networked organizations. In: 12th Enterprise Distributed Object Computing Conference Workshops, pp. 276-287. IEEE (2008)

24. Schekkerman, J.: Extended enterprise architecture maturity model support guide, version 2.0. Institute for Enterprise Architecture Developments (2006)

25. Thomas, R., Tiemann, M., Hite, R., McVay, W., Brundage, G., Knuth, D., Paradis, P., Cullen, T.: Architecture alignment and assessment guide, pp. 1-39. CIO Council (2000)

26. UNDESA. United nations global e-government readiness report 2004: Toward access for opportunity. Technical report (2004)

27. United State Office of Personal Management. Structured interviews: A practical guide

28. Yin, R.: Case study research: Design and methods, vol. 5. Sage publications, Inc. (2009) 